Revue des patrimoines

\title{
Un service diocésain de la conservation du patrimoine : l'exemple du diocèse de Cambrai (Nord)
}

\section{Caroline Biencourt}

\section{(2) OpenEdition}

\section{Journals}

Édition électronique

URL : http://journals.openedition.org/insitu/6026

DOI : $10.4000 /$ insitu. 6026

ISSN : 1630-7305

Éditeur

Ministère de la culture

\section{Référence électronique}

Caroline Biencourt, «Un service diocésain de la conservation du patrimoine : l'exemple du diocèse de Cambrai (Nord) », In Situ [En ligne], 11 | 2009, mis en ligne le 18 avril 2012, consulté le 19 avril 2019. URL : http://journals.openedition.org/insitu/6026 ; DOI : 10.4000/insitu.6026

Ce document a été généré automatiquement le 19 avril 2019

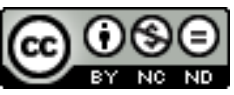

In Situ Revues des patrimoines est mis à disposition selon les termes de la licence Creative Commons Attribution - Pas d'Utilisation Commerciale - Pas de Modification 4.0 International. 


\title{
Un service diocésain de la conservation du patrimoine : l'exemple du diocèse de Cambrai (Nord)
}

\author{
Caroline Biencourt
}

1 Dans son allocution du 12 octobre 1995 aux membres de la première assemblée plénière de la Commission pontificale pour les biens culturels de l'Église ${ }^{1}$, Jean-Paul II définit la notion de «biens culturels» de l'Église de la façon suivante : il s'agit des «patrimoines artistiques de la peinture, de la sculpture, de l'architecture, de la mosaïque et de la musique mis au service de la mission de l'église. Il faut ajouter à tout cela les biens sous forme de livres, qui se trouvent dans les bibliothèques ecclésiastiques, et les documents historiques conservés dans les archives des communautés ecclésiales $»^{23}$.

2 En Italie, ce concept paraît assimilé, contrairement à la France. En effet, aux niveaux diocésains, deux entités indépendantes ont traditionnellement la responsabilité de ces «biens culturels ». Sans généraliser, on trouve d'une part des commissions d'art sacré ${ }^{4}$ qui ont avant tout un rôle dans la conception et la réalisation de projets d'aménagements liturgiques dans les édifices religieux et d'autre part, des archivistes qui veillent sur les archives dites « historiques ». Parfois, des commissions d'archives et d'histoire tentent de réunir ces deux pôles, tant bien que mal.

3 À Cambrai, le problème de la gestion d'un aussi vaste ensemble patrimonial s'est posé quasiment tout de suite après la Première Guerre mondiale et la reconstruction. En effet, dès 1926, a été créée une Commission d'histoire religieuse et d'art sacré. Présenter son histoire permet de comprendre comment elle a préfiguré le service diocésain du patrimoine culturel institué en 2003. Après avoir décrit les missions et le fonctionnement de cette nouvelle structure, un bilan de ses activités à court terme peut être dressé. De nouveaux chantiers d'intervention se dessinent et laissent entrevoir quelques perspectives d'avenir. 


\section{Historique}

4 Mgr Jean-Arthur Chollet ${ }^{5}$ instaure en 1926, une Commission d'histoire religieuse et d'art sacré ${ }^{6}$ ayant pour but :

- de conserver des archives mais aussi des objets,

- d'inventorier les archives paroissiales et d'accepter d'éventuels versements,

- de contribuer à la recherche scientifique.

Dès sa création, les domaines d'intervention de la commission sont très larges et associent déjà à la gestion du patrimoine écrit celle du patrimoine mobilier, sans oublier d'encourager les études historiques.

\section{Figure 1}

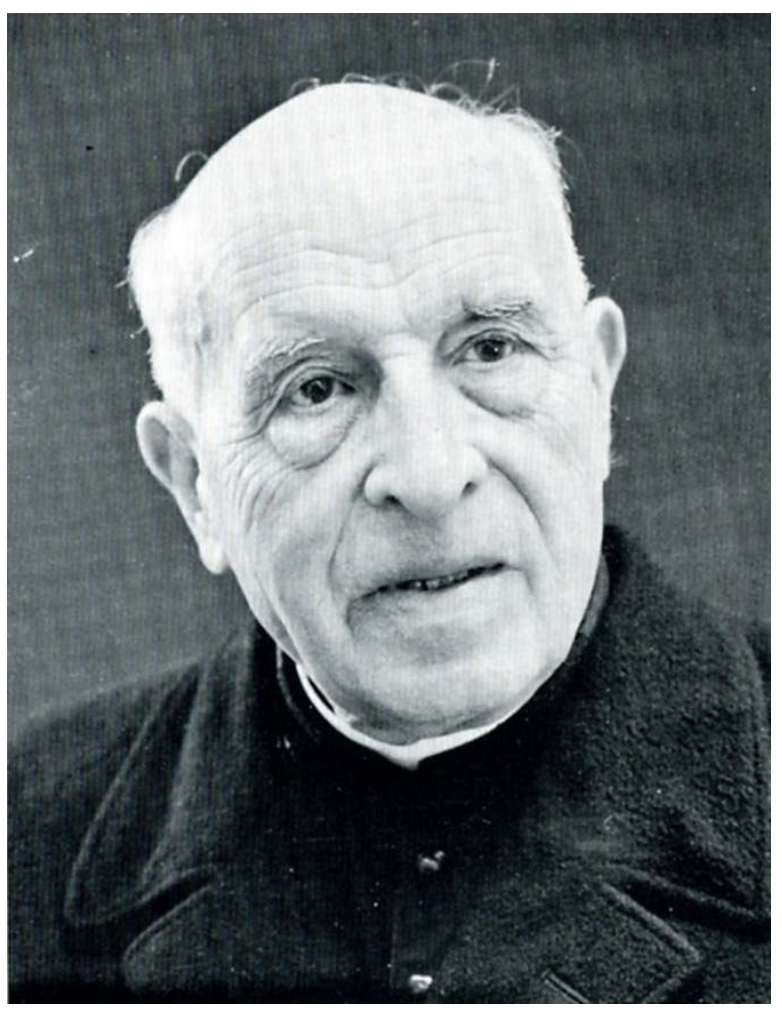

Portrait de Cyrille Thelliez par M. Delcroix publié dans SOCIÉTÉ D'ÉMULATION DE CAMBRAI. Un historien cambrésien. Le chanoine Cyrille Thelliez (1888-1970). IN MEMORIAM, Cambrai-Paris, [1970] Phot. Caroline Biencourt, 2008. ( ) Diocèse de Cambrai

6 Un jeune prêtre, Cyrille Thelliez ${ }^{7}$, considéré par ses professeurs du grand séminaire comme un rat de bibliothèque féru d'histoire, est désigné secrétaire. Vingt ans plus tard, alors qu'il assure toujours cette fonction, il est nommé archiviste diocésain et conservateur du Musée diocésain ${ }^{8}$ dont il est le fondateur. En effet, il s'était évertué à rassembler de nombreux objets religieux provenant du diocèse. La conception et la réalisation de ce musée sont une des œuvres de sa vie ${ }^{9}$. En 1958, premier musée d'art religieux ouvert en France, il avait un statut provincial, correspondant aux trois diocèses de Cambrai, Lille et Arras $^{10}$. 
7 En 1967, Mgr Jenny ${ }^{11}$ remanie la commission sans modifier ses attributions ${ }^{12}$. En la renommant Commission d'histoire du diocèse, il souhaite donner un nouvel élan en désignant de nouveaux membres parmi lesquels on trouve mon prédécesseur, le Père Félicien Machelart. Le chanoine Thelliez en fait encore partie en tant qu'archiviste diocésain.

Pendant quarante-quatre ans, la gestion du patrimoine culturel fut confiée à un seul prêtre, l'abbé Thelliez. À son décès, en 1970, le Père Machelart lui succède comme archiviste mais, dès 1973, il cumule ce poste avec celui de secrétaire de la Commission diocésaine d'art sacré dont il devient finalement le responsable délégué quelques années plus tard.

9 Jusqu'en 2001, date de mon entrée en fonction pour le diocèse de Cambrai, la situation ne connut aucun changement. Initialement chargée des archives $^{13}$, j'ai été associée par la force des choses au secrétariat de la Commission diocésaine d'art sacré ainsi qu'à la gestion des réserves du Musée diocésain.

10 Étant donné la réalité du terrain (liens ténus entre archives, musée et Commission diocésaine d'art sacré) et du fait des orientations de la Commission pontificale des biens culturels, le Père Machelart souhaitait la création d'un service de la conservation du patrimoine chargé de la gestion des biens culturels du diocèse. En 2003 naissait le service diocésain du patrimoine culture ${ }^{14}$ qui apparaissait comme tel, pour la première fois, dans l'Annuaire diocésain ${ }^{15}$.

\section{Missions du service}

Elles sont présentées ainsi dans l'Annuaire diocésain ${ }^{16}$ :

1. conservation et traitement des archives diocésaines et paroissiales,

2. gestion de la bibliothèque historique, annexe des archives,

3. sauvegarde et conservation préventive du patrimoine mobilier et immobilier affecté au culte ou propriété du diocèse,

4. gestion du Musée diocésain.

12 Afin d'être plus précis, il est possible de scinder le patrimoine culturel du diocèse de Cambrai en deux catégories :

- le patrimoine écrit: archives (historiques et courantes), bibliothèques, registres de catholicité,

- le patrimoine mobilier et immobilier : les réserves du musée, les objets, conservés ou non dans les églises communales, affectés au culte.

13 Il faut noter qu'à ces tâches s'ajoute toujours celle du secrétariat de la Commission diocésaine d'art sacré.

\section{Identification}


Figure 2

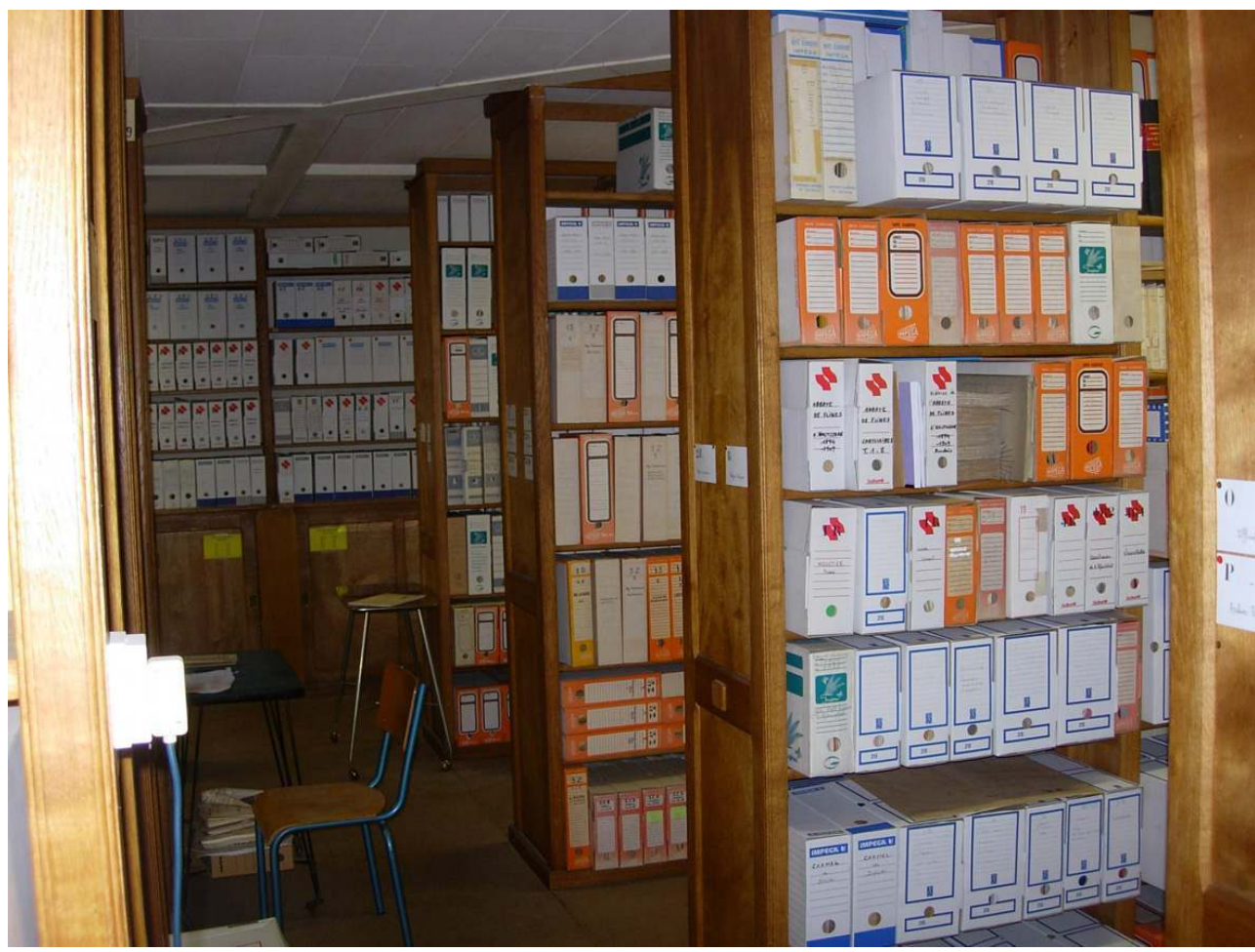

Un des magasins d'archives

Phot. Caroline Biencourt, 2008. (c) Diocèse de Cambrai

14 La gestion du patrimoine écrit et mobilier à caractère culturel échoit donc au responsable $\mathrm{du}$ Service diocésain du patrimoine culturel, appelé conservateur diocésain du patrimoine. S'inspirant des titulatures employées par l'État, cette dénomination a été choisie pour des raisons effectives ainsi que par commodité.

En effet, la préoccupation première est de "conserver " l'ensemble du patrimoine, propriété du diocèse ou affecté au culte, de quelque nature qu'il soit (du papier aux œuvres d'art). De plus, l'appellation «conservateur diocésain du patrimoine» est plus commode et plus compréhensible que celle de «responsable du service du patrimoine culturel du diocèse ».

Ce changement de désignation correspond à une volonté d'institutionnaliser un service qui existait déjà de fait. C'est finalement la solution médiane entre une terminologie romaine, pas encore véritablement assimilée par les structures religieuses françaises, et une appellation plus explicite sur les missions jusque-là assurées par l'archiviste diocésain et qui dépassaient très largement le domaine des archives.

Il faut reconnaître que ce nouveau titre assoit l'autorité du conservateur diocésain. Il permet d'abord de faire respecter plus facilement les droits de l'affectataire, ensuite de faire prendre conscience à ce dernier de ses devoirs en matière de patrimoine culturel et, en conséquence, de les lui faire appliquer.

18 Par ailleurs, cette initiative est plutôt bien accueillie et perçue au niveau local et régional par les représentants du secteur public avec lesquels le service diocésain est couramment en relation (le conservateur régional des monuments historiques, le service de 
l'inventaire général, la direction régionale des affaires culturelles, le conservateur des antiquités et objets d'art, etc.)

\section{Fonctionnement}

19 À l'heure actuelle, le service ne dispose que d'une personne pour assurer toutes les tâches : l'aide de bénévoles serait bienvenue.

\section{Trouver sa place et créer une dynamique}

Dans un premier temps, il est nécessaire de sensibiliser des partenaires potentiels (diacres, curés, animateurs en pastorale, laïcs en responsabilité, mouvements et services d'Église, etc.) aux actions du service du patrimoine. À titre d'exemple, les interventions dans les paroisses pour récupérer des archives en péril ou pour initier des procédures de collecte permettent de rencontrer de nouveaux interlocuteurs et de créer une dynamique diocésaine. Il est effectivement utile de pouvoir compter directement sur des bénévoles qui acceptent de s'occuper des archives localement.

La création d'un réseau ne doit pas s'établir seulement en interne mais aussi s'inscrire dans le tissu culturel déjà existant. Ainsi, le démarchage auprès d'universités proposant des formations professionnalisantes est vital pour un service comme le nôtre. D'abord parce qu'il informe de notre existence et parce qu'il présente nos besoins. Incontestablement, l'accueil d'étudiants peut faire progresser les projets d'inventaire et de catalogage pour mettre à disposition des chercheurs, amateurs et professionnels, de nouvelles sources historiques, encore inédites.

\section{Appréhender l'ampleur des missions}

22 Les activités qui incombent au responsable du service sont multiples, le danger de s'y perdre est réel. Accepter le fait qu'il était impossible de tout mener de front et de régler rapidement les problèmes en attente a pris du temps : il a fallu planifier les chantiers et établir des priorités. Ce n'est pas pour autant que la réalisation du programme ne prend pas de retard. Répondre aux besoins impromptus et urgents qui s'imposent assez régulièrement (tri de papiers ou d'une bibliothèque lors du décès d'un prêtre, fermeture d'établissement, mise en sécurité d'objets, projet diocésain, etc.) rentre naturellement dans ses attributions.

Même si le travail est considérable, il n'est pas pour autant insurmontable. Le récolement des collections du Musée diocésain nécessite une préparation minutieuse (élaboration d'un programme d'application, identification des besoins en main d'œuvre supplémentaire, etc.) si l'on ne veut pas y perdre de temps.

\section{Prendre du recul}

La multiplicité des domaines d'action peut être considérée comme un réel inconvénient ; on peut voir les choses différemment. En effet, appréhender le patrimoine dans son ensemble permet de rendre à chaque catégorie la place qui est la sienne et de la replacer 
dans l'ensemble. Cette approche globale est l'occasion de découvrir les ressources patrimoniales du diocèse, d'étendre ses connaissances et de les approfondir.

\section{Bilan d'activité}

Au bout d'un an dans ce service ainsi redéfini, voici les deux principaux changements notables qu'il est possible d'observer.

\section{Une ouverture}

Après avoir longtemps manqué de visibilité et d'identité, le service diocésain du patrimoine culturel est désormais connu et reconnu non seulement par les acteurs diocésains mais aussi par les partenaires culturels extérieurs (municipalités, conservateurs de musées, universités, archives départementales et municipales, etc.)

27 Tisser des liens était une priorité pour faire exister le service et lui donner du sens; aujourd'hui, le conservateur diocésain du patrimoine apparaît comme un interlocuteur compétent et qualifié sur l'échiquier culturel local et régional.

\section{Une réussite à conforter}

Le service est de plus en plus sollicité, surtout par les paroisses, pour la collecte d'archives, pour la mise en œuvre du guide d'archivage ou encore pour des conseils en matière de conservation dans les églises.

Lors de rencontres in situ, les besoins et les demandes sont très clairement énoncées. Les paroissiens comme les municipalités expriment fréquemment le désir de se réapproprier ce patrimoine, de le sauvegarder et enfin, de le valoriser. Ils attendent ainsi du service diocésain qu'il puisse les aider dans ces démarches.

\section{Perspectives}

Ces réflexions laissent entrevoir de futurs chantiers encore plus vastes que ceux qui sont déjà en cours.

\section{Organisation rationnelle des collections patrimoniales, à l'échelle du diocèse}


Figure 3

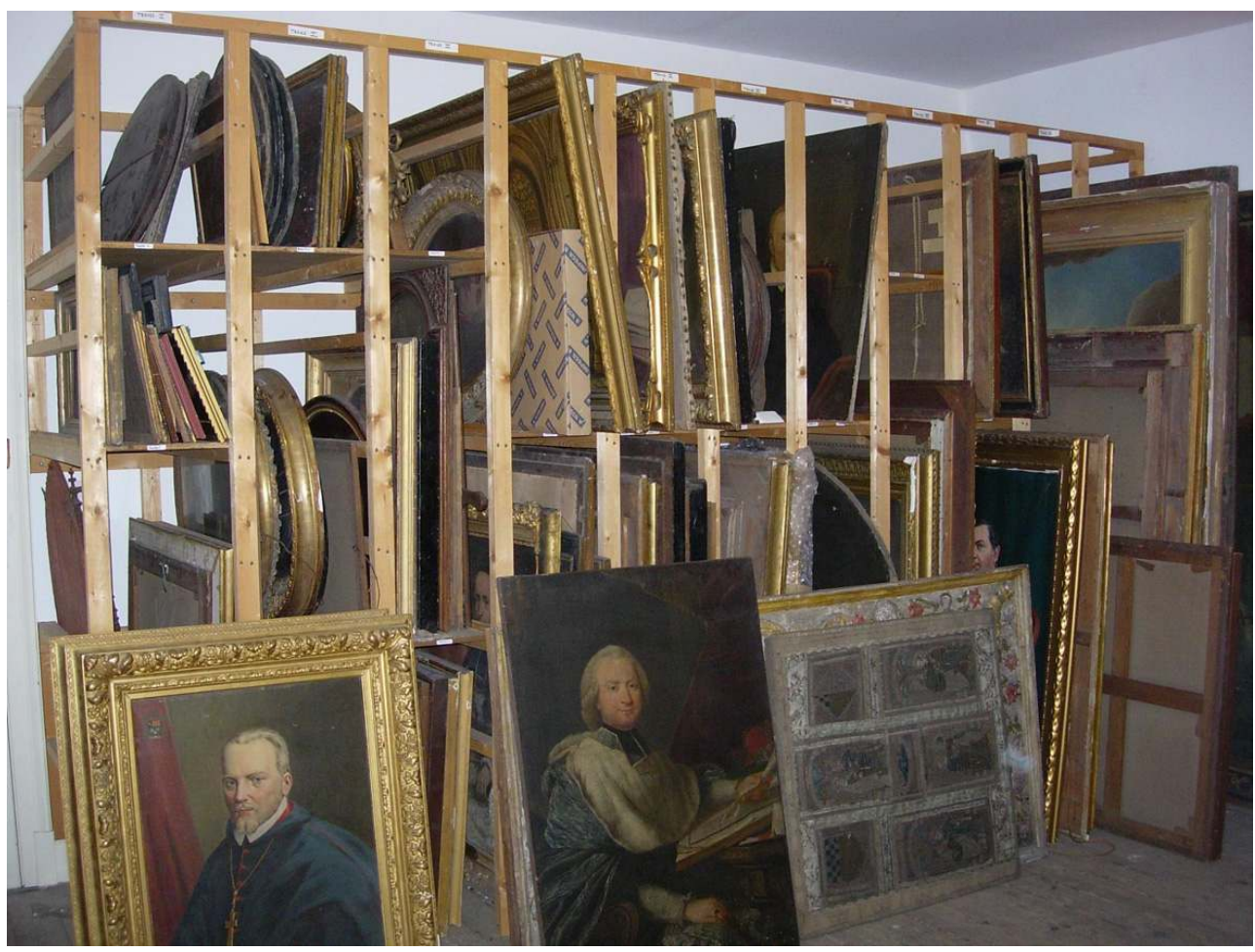

Râtelier des réserves du musée diocésain

Phot. Caroline Biencourt, 2008. (c) Diocèse de Cambrai

31 Le service est actuellement confronté à l'accumulation massive d'archives, d'objets ou de vêtements liturgiques, etc. apportés régulièrement par des paroissiens, qui saturent les locaux. Les causes de ces récupérations systématiques sont le plus souvent :

- le débarras de bâtiments ou de presbytères lors de ventes ou de rétrocessions aux communes $^{17}$,

- le principe de précaution : des travaux vont avoir lieu dans l'église ou dans un presbytère et tout ce qui est considéré comme « très important » est mené à l'archevêché,

- le «ménage » des sacristies.

Cette collecte non sélective n'est pas envisageable: refuser le tri est une position déraisonnable. En archivistique, les tris sont facilités par l'application de tableaux de gestion $^{18}$. En revanche, dans le domaine des objets, cela est plus difficile mais faisable.

La connaissance des fonds est une nécessité. Elle passe par la réalisation d'inventaires, travail essentiel à mener conjointement par les propriétaires et les affectataires. À partir de ces outils, des solutions adéquates de traitement pourront être proposées. Les choix définis limiteront alors les erreurs, notamment en matière d'élimination, risque assez important à l'heure actuelle.

\section{Sécurisation des objets d'art dans les églises}

Donner des conseils en matière de sécurisation des objets mobiliers dans les lieux de culte est devenu assez complexe. Le vieux réflexe, souvent lié aux traumatismes de vols ou de 
dégradations, est de fermer les églises, mettre les objets à l'abri dans des «lieux plus sûrs » (souvent chez des personnes privées) ou encore faire des copies d'œuvres.

Il est indispensable de faire évoluer les esprits et de les convaincre que d'autres solutions sont préférables : procéder à un inventaire, vérifier la sécurité du lieu et assurer un gardiennage, même ponctuel.

De plus, on peut estimer qu'il est du devoir de l'affectataire d'alerter les propriétaires quand les conditions de conservation sont ou deviennent précaires. Cette démarche vise évidemment à sauver des objets ou des documents d'une destruction inéluctable à court, moyen ou long terme. Dans ce cas, des actions conduites avec le propriétaire, ou du moins avec son accord, doivent être mises en œuvre.

\section{Formation des interlocuteurs}

La bonne volonté des paroissiens n'est pas toujours compatible avec les principes de conservation préventive. Il est regrettable que leur souci de bien faire conduise à des erreurs par ignorance ou manque de précautions.

Figure 4

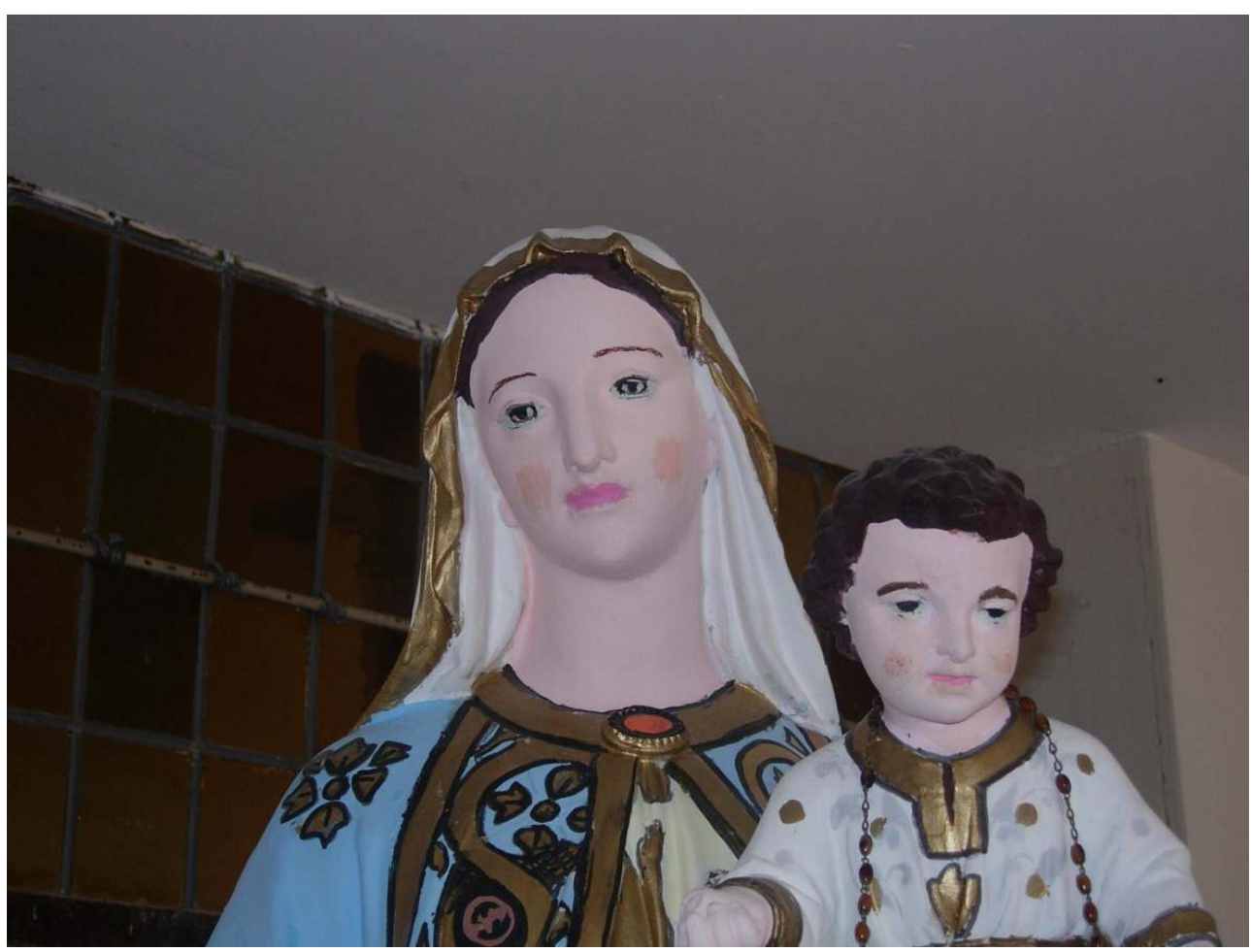

Exemple de repeint malheureux sur une statue en plâtre d'une Vierge à l'enfant

Phot. Caroline Biencourt, 2008. (c) Diocèse de Cambrai

Ce constat pose la question de la formation de ces interlocuteurs déjà sensibilisés au patrimoine, par leur implication dans la sauvegarde des objets mobiliers ou des archives. Les simples conseils dispensés au cours d'une visite ne sont pas suffisants : des initiations à la conservation préventive pourraient être une orientation à retenir. 
39 En 2009, pour la première fois dans notre diocèse, une journée autour de l'art sacré sera organisée par la Commission diocésaine d'art sacré ${ }^{19}$, au cours de laquelle cet aspect sera probablement abordé.

\section{Valorisation du patrimoine mobilier}

Des inventaires sérieux sont les outils les plus utiles pour valoriser les objets du culte. En s'appuyant sur le travail réalisé, il est plus facile de répondre à des demandes d'exposition et de participer à des manifestations culturelles.

41 Le diocèse de Cambrai a vécu cette année un événement rarissime : la célébration d'un millénaire ${ }^{20}$. Un des projets était de concevoir un espace de type muséographique ${ }^{21}$ pour présenter historiquement et pastoralement mille ans de dévotion mariale. Grâce à un inventaire détaillé, il a été possible de sélectionner des œuvres répondant au programme scientifique.

\section{Dimension pastorale}

Le service du patrimoine culturel commence à prendre dans le diocèse une dimension pastorale qui n'est pas évidente de prime abord. Pourtant, les objets mobiliers et les archives sont des preuves matérielles de la vie religieuse et de l'attachement à la foi.

On ne visite pas une église comme un musée : une église est par essence un édifice cultuel. Cette dimension est fondamentale. À partir de l'histoire d'un édifice, des dévotions et pratiques populaires qui lui sont attachées, de son décor, on peut bâtir un discours pastoral, voire purement catéchétique. Associé à la Commission diocésaine d'art sacré, le service du patrimoine devrait pouvoir aider les communautés locales à redécouvrir leur patrimoine en leur donnant de nouvelles clés de lecture. 


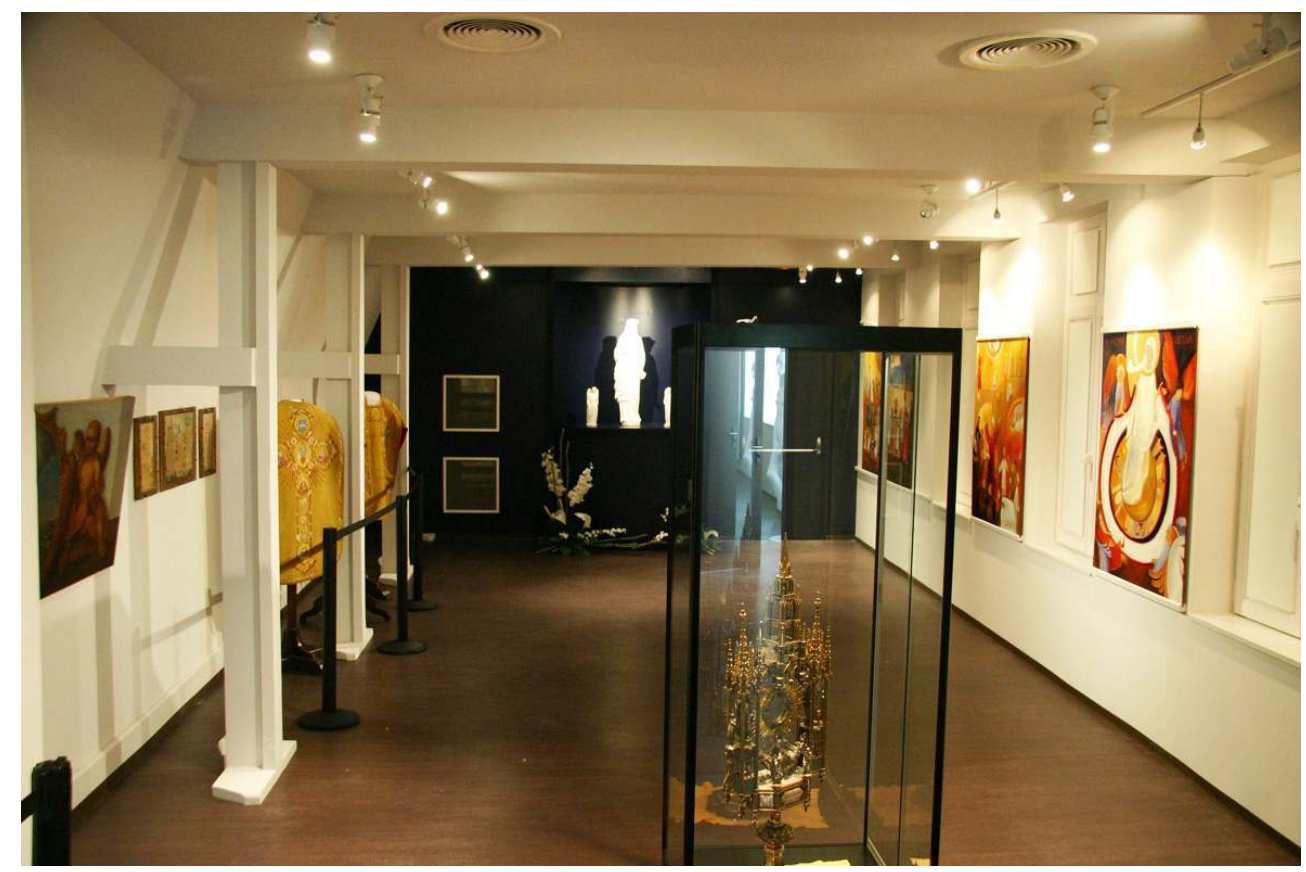

Vue de l'espace Bertholin, rue Abel de Pujol à Valenciennes

Phot. Bruno Feillet, 2008. @ Diocèse de Cambrai

Le service du patrimoine culturel du diocèse de Cambrai a eu une genèse difficile mais a enfin trouvé son identité. Sa création répond essentiellement aux besoins internes et externes qui se font sentir en matière de sauvegarde des biens patrimoniaux, qu'il s'agisse de patrimoine écrit, mobilier ou immobilier. Évoluant vers un «service diocésain de la conservation du patrimoine ", fort de ses compétences, son rayonnement dépasse peu à peu la sphère ecclésiale diocésaine.

Ses domaines d'intervention sont très étendus, mais cette polyvalence contribue à une meilleure connaissance générale $d u$ patrimoine religieux dans un vaste territoire géographique.

Une des préoccupations du service est d'établir des procédures qui visent à modifier des habitudes et à encadrer l'application de nouveaux choix de conservation.

L'expérience acquise permet d'envisager de nouveaux projets ambitieux et de nouvelles orientations : les questions sont celles du temps et des ressources disponibles.

\section{NOTES}

1. Documents en ligne de la commission pontificale des biens culturels de l'Église: http:// www.vatican.va/roman_curia/pontifical_commissions/pcchc/documents/rc_com_pcchc_indexdocuments_fr.html. 
2. Jean-Paul II. L'importance du patrimoine artistique dans l'expression de la foi et le dialogue avec l'humanité, La Documentation catholique, 19 novembre 1995, n²126, p. 969-971.

3. Voir également sur le sujet, dans ce numéro, l'article d'Alain Nafilyan : La reconstruction des édifices religieux en Basse-Normandie après la Seconde Guerre mondiale.

4. Commission épiscopale de liturgie et de pastorale sacramentelle et du Comité national d'Art Sacré. Les responsabilités des Commissions diocésaines d'Art Sacré. Orientations et règles de travail, 8 octobre 1999. Voir sur le site: http://www.liturgiecatholique.fr/IMG/pdf/ Resp_des_commissions_diocesaine.pdf [date de consultation : 08/09/08]. Site Internet du Service national de la pastorale liturgique et sacramentelle : http://www.liturgiecatholique.fr/-Servicenational-de-la-pastorale-.html

5. Mgr Jean-Arthur Chollet (1862-1952), archevêque de Cambrai de 1913 à 1952.

6. Mgr Chollet, Jean-Arthur. Ordonnance du 13 février 1926 instituant une commission d'histoire religieuse et d'art sacré, Semaine religieuse du diocèse de Cambrai, 27 février 1926, nº 9 , p. 101-102. L'article 2 porte sur ses missions, l'article 3 sur les locaux et l'article 4 sur les nominations des membres. Voici en totalité le contenu de l'article 2: «Art. 2: Elle aura pour mission de veiller à la conservation des documents manuscrits ou imprimés appartenant à cette histoire et des monuments ou objets d'art religieux. Elle en fera l'examen et l'inventaire dans les paroisses et en contrôlera la garde. Elle classera et recueillera ceux qui lui seront offerts. Elle provoquera des études d'histoire et d'art chrétiens. Elle guidera les recherches, elle conseillera les auteurs de ces études et recherches. Elle fera prendre des copies des documents anciens intéressant l'histoire du diocèse et dont elle ne pourra pas se procurer les originaux. Elle s'inspirera du code J.C. et des instructions du Saint- Siège ».

7. Cyrille Thelliez (1888-1970) est ordonné prêtre du diocèse de Cambrai en 1920. Licencié en histoire, il devient membre de la Société d'émulation de Cambrai en 1912. Sa passion pour l'histoire lui vaudra d'assurer des fonctions culturelles au sein du diocèse (secrétaire de la Commission d'histoire et d'art sacré, archiviste, conservateur du Musée diocésain) en plus de sa charge de curé de paroisse. Auteur de plusieurs ouvrages historiques et de monographies, il recevra notamment pour celui intitulé La merveilleuse image de Notre-Dame de Grâce de Cambrai le prix Louis Danel de la Société des sciences de l'agriculture et des arts de Lille, spécialité Histoire. L'un de ses plus grands projets fut la création d'un musée diocésain d'art sacré en 1956, installé officiellement dans la chapelle du grand séminaire de Cambrai (chapelle des Jésuites) en 1958.

8. La Quinzaine diocésaine de Cambrai, 15 novembre 1946, nº 4, p. 38 : «L'abbé Cyrille Thelliez, curé de Mastaing, est nommé conservateur du Musée diocésain et archiviste diocésain ».

9. Avant d'être installé dans la chapelle des Jésuites, le musée d'art se situait dans la salle du tribunal révolutionnaire. $\mathrm{Y}$ étaient présentées de nombreuses œuvres d'art (peintures, sculptures, différents objets religieux, ...) qu'il avait lui-même rassemblées. Elles provenaient des paroisses du diocèse et de collections particulières, dont celle de Mgr Chollet.

10. Labellisé Musée de France, il est fermé au public depuis 1980.

11. Mgr Henri-Martin Jenny (1904-1982), archevêque de Cambrai de 1966 à 1980.

12. Mgr Jenny. Ordonnance instituant la commission d'histoire du diocèse, La Quinzaine diocésaine de Cambrai, 6 janvier 1967, nº 1, p. 5. Les tâches de cette nouvelle commission sont « de garder le souvenir des événements passés par la recherche des faits et des documents, leur conservation sous toutes ses formes, de faire mieux connaitre la vie actuelle du diocèse ; elle contribuera ainsi, à sa façon, d'une part à la culture intellectuelle (...) et d'autre part à une pastorale d'Église (...) ».

13. Embauchée pour être "archiviste adjoint ", mon statut était celui de "bibliothécaire », seul reconnu parmi ceux du personnel laïc de l'Église de France. À l'heure actuelle, les statuts ne citent toujours pas la fonction d'archiviste et encore moins celle de conservateur diocésain du patrimoine. 
14. Site Internet $d u$ service $d u$ patrimoine culturel diocésain: http:// archives.cathocambrai.com/.

15. Dans l'édition de l'Annuaire diocésain de 2003 (p. 63), les charges du service du patrimoine culturel du diocèse sont présentées ainsi : « - archives historiques du diocèse et des paroisses ; bibliothèques et musée diocésain ; - commission d'art sacré ; - objets affectés au culte ».

16. Annuaire diocésain de Cambrai, édition 2008, p. 69.

17. Jusqu'alors, l'archiviste était rarement informé des mutations immobilières et voyait arriver en masse des documents ou des objets. Grâce aux échanges avec le service immobilier, des interventions sur place sont aujourd'hui programmées pour procéder aux tris.

18. J'ai mis au point un guide d'archivage pour aider les paroisses à trier leurs papiers et prévoir l'archivage définitif de documents importants. Cette procédure de rationalisation vise à modifier les usages « radicaux » : élimination ou conservation intégrales des fonds.

19. Pour la concevoir, nous nous inspirons bien évidemment d'initiatives d'autres diocèses.

20. Depuis 1000 ans, chaque année, les Valenciennois célèbrent la Vierge qui a intercédé pour les délivrer de la peste. En déployant un Saint-Cordon autour de l'enceinte de la ville, ils furent exaucés.

21. Inauguration de la Maison du pèlerin à Valenciennes à l'occasion des fêtes du Millénaire du Miracle du Saint-Cordon et présentation de la salle d'exposition: http:// www.cathocambrai.com/page_ln-50782.html. L'exposition « Les merveilles du Saint-Cordon » est visible dans la Maison du pèlerin, 21, rue Abel de Pujol à Valenciennes (Nord). Espace ouvert tous les mardis et vendredis de $10 \mathrm{~h}$ à $12 \mathrm{~h}$ et de $14 \mathrm{~h} 30$ à $17 \mathrm{~h} 30$. Pour tout renseignement, tél. 0327462020 ou e-mail : espace-bertholin@orange.fr.

\section{RÉSUMÉS}

Dans le diocèse de Cambrai a été instauré un service diocésain du patrimoine culturel dont la mission est la gestion des patrimoines écrits, mobiliers et immobiliers, propriété de l'association diocésaine ou affecté au culte. Ce retour d'expérience permet de présenter le sens, l'intérêt et les enjeux d'une telle responsabilité en terme de connaissance, de conservation et de valorisation d'un aussi vaste ensemble patrimonial cohérent.

The diocese of Cambrai has recently set up its own heritage service. The missions of this service include the conservation of the written heritage of the diocese, its mobile and built heritage and in general the property of the diocesan association or belonging otherwise to the church. The article relates the experience of this service and the interest of its responsibilities in terms of the understanding, the conservation and the interpretation of an extensive but coherent heritage. 


\section{INDEX}

Keywords : Religious heritage, Management of church heritage, Liturgical furnishings, Ecclesiastical archives, Diocesan archives, Parish archives, Cultural heritage of the diocese of Cambrai, Pontifical commission for the church's cultural properties, Commission for sacred art, Commission for religious history and sacred art, Diocesan service of cultural heritage, Commission for the history of the diocese of Cambrai, Diocesan Commission for sacred art, Diocesan archivist, Diocesan heritage curator, Cambrai diocesan museum, Museum of religious art

Mots-clés : Musée diocésain de Cambrai, musée d'art religieux, archiviste diocésain, conservateur diocésain du patrimoine, Jean-Paul II, Mgr Jean-Arthur Chollet, Cyrille Thelliez, Mgr Jenny, Félicien Machelart, diocèse de Cambrai, gestion du patrimoine religieux, gestion du patrimoine de l'Église, aménagements liturgiques, archives ecclésiastiques, archives diocésaines, archives paroissiales, patrimoine culturel du diocèse de Cambrai, Commission pontificale pour les biens culturels de l'Église, commissions d'art sacré, Commission d'histoire religieuse et d'art sacré, Service diocésain du patrimoine culturel, Commission d'histoire du diocèse de Cambrai, Commission diocésaine d'Art sacré

\section{AUTEUR}

\section{CAROLINE BIENCOURT}

Conservateur diocésain du patrimoine, diocèse de Cambrai. archivistecambrai@nordnet.fr 